\title{
Electronic Structure of Small Fullerenes: Evidence for the High Stability of $\mathbf{C}_{\mathbf{3 2}}$
}

\author{
H. Kietzmann, R. Rochow, G. Ganteför, and W. Eberhardt \\ Forschungszentrum Jülich GmbH, Institut für Festkörperforschung, D-52425 Jülich, Germany \\ K. Vietze and G. Seifert \\ Institut für Theoretische Physik, Technische Universität Dresden, D-01062 Dresden, Germany \\ P. W. Fowler \\ Department of Chemistry, University of Exeter, Stocker Road, Exeter EX4 4QD, United Kingdom
}

\begin{abstract}
The electronic structure of small fullerenes determined experimentally by anion photoelectron spectroscopy is compared with calculations. A huge mass signal and a large gap of $1.3 \mathrm{eV}$ comparable to the gap of $\mathrm{C}_{70}$ have been found for $\mathrm{C}_{32}$, indicating that this cluster is the most stable fullerene below $\mathrm{C}_{60}$. Also $\mathrm{C}_{36}, \mathrm{C}_{44}$, and $\mathrm{C}_{50}$ exhibit large gaps and surprisingly high stabilities. The criteria for the selection of clusters sufficientl stable to form a cluster material are discussed.
\end{abstract}

PACS numbers: 71.20.Tx, 36.40.-c, 71.24.+q, 79.60.-i

Cluster materials add a new dimension to materials science. The building blocks of such materials are stable clusters, i.e., small particles containing a well-define number of atoms or molecules. They can be condensed into bulk materials while keeping their individual identity, if the intracluster covalent bonding is stronger than the van der Waals cluster-cluster bonding. The most famous example is $\mathrm{C}_{60}$, a "soccer ball" of 60 carbon atoms. Two $\mathrm{C}_{60}$ interact only weakly and have a considerable energy barrier to spontaneous coalescence. Therefore, although bulk graphite is the energetically most stable form of bulk carbon, crystals made of $\mathrm{C}_{60}$ are highly stable and form a new metastable phase of carbon in addition to diamond.

One criterion for a cluster to be suitable to form a cluster material is, apart from its abundance, the height of the energy barrier for chemical reactions with identical clusters and with the substances used in the process of extraction and separation. Probably, for any cluster with an electronically closed shell such a barrier will exist. However, if it is too small, the clusters might merge into an amorphous bulk material even at room temperature. The barrier for possible reaction between two neighboring clusters may be correlated with the energy gap $\left(E_{\text {gap }}\right)$ between the electronic ground state and the firs excited state of the neutral cluster. For a free cluster, this quantity can be derived by anion photoelectron spectroscopy. We here propose to use it as a criterion to identify candidates for new cluster materials.

Beyond $n=30$, the fullerenes are the most stable carbon clusters $[1,2]$. Below $\mathrm{C}_{30}$ the cluster intensity in the mass spectra decreases drastically and the photoelectron spectra differ strongly from those of the fullerenes [1], indicating a change in stable structure near $\mathrm{C}_{30}$ as confirme by theory [2]. Cluster materials have been formed from $\mathrm{C}_{60}$ and larger fullerenes, but so far it has not been pos- sible to extract smaller fullerenes from the raw soot and no such fullerene materials have been synthesized. The question of suitable candidates for fullerene materials below $\mathrm{C}_{60}$ in mind, we have examined the electronic band gaps and the stabilities of the small fullerenes theoretically and experimentally.

The results shown in this Letter give strong indication that smaller fullerenes are indeed formed in a furnace under appropriate conditions and that some of these species are as stable as the fullerenes larger than $\mathrm{C}_{60}$. We present a systematic experimental and theoretical study of the gap energies $\left(E_{\text {gap }}\right)$ of small carbon fullerenes. The most prominent fullerene below $\mathrm{C}_{60}$ is $\mathrm{C}_{32}$ with an experimentally derived $E_{\text {gap }}$ of $1.3 \mathrm{eV}$, only slightly smaller than the gap of $\mathrm{C}_{60}\left(E_{\text {gap }}=1.6 \mathrm{eV}\right)$. The experimental gaps are in excellent agreement with the results of calculations. Additionally, the abundances of the clusters in the gas phase mass spectrum correlate with the stability with respect to fragmentation or growth. Thereby the loss or addition of $\mathrm{C}_{2}$ is considered as the relevant fragmentation or growth process as suggested by the results in [3] and [4]. Indeed, under the experimental conditions chosen for our cluster source, $\mathrm{C}_{32}$ is the most intense peak in the spectrum, even larger than the peak of $\mathrm{C}_{60}$, in agreement with a large stability calculated for $\mathrm{C}_{32}$.

The experimental setup has been described in detail previously [5]. Using a standard laser evaporation source, the yield of small fullerene anions with less than 60 atoms has been optimized by additional annealing. Immediately following the condensation process, the carbon clusters have been strongly heated in an electrical discharge within the carrier gas $(\mathrm{He})$ and slowly cooled to room temperature. Thus, high intensities of fullerenes between $n=32$ and $n=50$ could be obtained, with the largest peak observed for $\mathrm{C}_{32}$. Weaker annealing leads to mass 
spectra, as shown in [1], with high peaks for $\mathrm{C}_{50}$ and $\mathrm{C}_{44}$, but only low abundances for smaller fullerenes. A Wiley-McLaren-type time-of-fligh mass spectrometer has been used to record the mass spectra. To examine the electronic structure, a cluster bunch of definit mass was irradiated by a $6.4 \mathrm{eV}$ UV pulse from an $\operatorname{ArF}$ excimer laser. The energy of the photoelectrons was analyzed by means of a "magnetic-bottle"-type time-offligh electron spectrometer with an energy resolution better than $50 \mathrm{meV}$. The absolute value of the energy calibration is uncertain to within $\pm 0.1 \mathrm{eV}$.

Figure 1 displays a mass spectrum of $\mathrm{C}_{n}$ cluster anions for $n=4$ to 70 atoms. Two size ranges with significan intensity may be distinguished: (i) $n=10-18$ and (ii) $n=32-60$. As discussed in [1], the most stable structures are in (i) monocyclic rings and (ii) fullerenes. Because of the strong annealing, small fullerenes between $n=32$ and $n=50$ are predominantly generated, in accordance with the results in [3]. The peaks of the "magic numbers" $n=32,36,44$, and 50 are even higher than the peak of $\mathrm{C}_{60}$. Slightly increased intensities compared to their neighbors have been observed before for $\mathrm{C}_{36}, \mathrm{C}_{44}$, and $\mathrm{C}_{50}$ [3,6-9]. A giant mass signal for $\mathrm{C}_{32}$ and the huge dominance of $\mathrm{C}_{36}, \mathrm{C}_{44}$, and $\mathrm{C}_{50}$ after strong annealing as reported here have not been seen before. As expected, clusters with $n=56,60$, and 70 also show enhanced abundances. The high abundance of $\mathrm{C}_{60}{ }^{-}$in the mass spectra is generally attributed to its extraordinarily high fragmentation stability. Thus, the large intensities for clusters with $n=32,36,44$, and 50 are strong indications for a high stability.

Obviously, thermodynamic stability alone cannot explain the relative abundances of the fullerenes. If the binding energy/atom is the only important factor for stability, all of the fullerenes beyond $\mathrm{C}_{70}$ should be more abundant than $\mathrm{C}_{60}$, which is certainly not the case. Undoubtedly, stability with respect to fragmentation barriers is one of the key parameters for the creation and the rela-

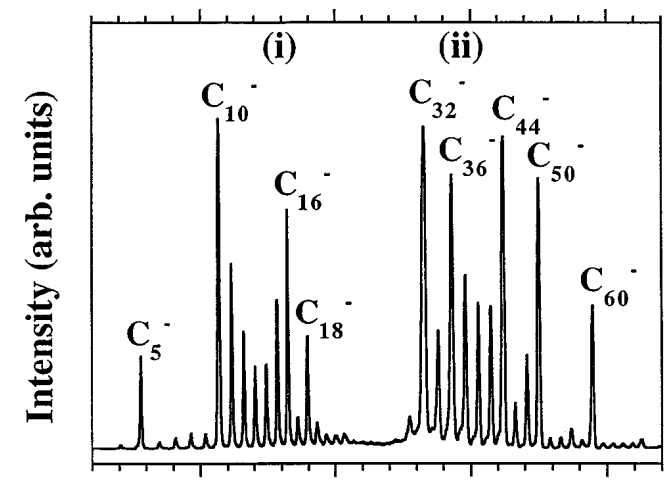

Time of flight (arb. units)

FIG. 1. Time-of-fligh mass spectrum of strongly annealed carbon cluster anions $\mathrm{C}_{n}{ }^{-}$. Size range (i): monocyclic rings; size range (ii): fullerenes. tive abundance of the fullerenes. However, to achieve a high abundance of a specifi fullerene $\mathrm{C}_{n}$, both a low stability of $\mathrm{C}_{n \pm 2}$ and a high stability of $\mathrm{C}_{n}$ against $\mathrm{C}_{2}$ loss or addition is required. The stability of the fullerenes with respect to fragmentation and growth may be described by a quantity $\delta$ that relates the energies of $\mathrm{C}_{n+2}$ and $\mathrm{C}_{n-2}$ to the energy of $\mathrm{C}_{n}$. If $E(n)$ is the total energy for $\mathrm{C}_{n}$, then

$$
\delta=E(n+2)+E(n-2)-2 E(n) .
$$

In previous calculations of the cluster stability [10] only the fragmentation was taken into account, not the possibility of further growth of the clusters.

Stability with respect to activated complexes of chemical reactions with identical clusters and with substances used for the extraction and isolation of fullerenes from soot is extremely important for the formation of fullerene materials. In the sense of stability against further fragmentation or addition reactions it is a key parameter for the relative abundances of the fullerenes [11]. Usually it is assumed that low chemical reactivity is associated with a large $E_{\text {gap }}$, because it is energetically unfavorable to extract electrons from a low lying HOMO (highest occupied molecular orbital) or to add them to a high lying LUMO (lowest unoccupied molecular orbital), which would be necessary to activate a reaction. Therefore suitable candidates for the formation of cluster materials are distinguished by high intensities in the mass spectrum and large gaps.

In Fig. 2, photoelectron spectra of selected carbon cluster anions beyond $n=30$ are presented. From these spectra, $E_{\text {gap }}$ can be directly extracted for clusters with a closed electronic shell configuratio [12]. The closed shell character of the neutral cluster leads to a singly occupied LUMO for the corresponding cluster anion. In the photoemission spectrum of $\mathrm{C}_{60}{ }^{-}$, peak $A$, as in the work by Yang et al. [13], is assigned to the emission of this electron from the LUMO. Thus it corresponds to the transition from the ground state of the anion to the ground state of neutral $C_{60}$. The second peak, $B$ of [13], is split by $0.3 \mathrm{eV}$ into two peaks $\left(B_{1}, B_{2}\right)$ here, due to the better resolution. In a one electron picture, peak $B$ is due to emission from the uppermost occupied orbitals of the HOMO leaving the cluster in its lowest electronically excited state $\left(h_{u}^{9} t_{1 u}^{1}\right)$. The observed splitting of peak $B$ could be the singlet-triplet splitting of this state, which has been found to be $0.24 \mathrm{eV}$ for $\mathrm{C}_{60}$ dissolved in methylcyclohexane [14]. In this case, a higher intensity of the triplet $B_{1}$ as compared to the singlet $B_{2}$ would be expected, although this is not found in our spectra. With this interpretation of peak $B$, the separation of the two features $A$ and $B$ corresponds to $E_{\text {gap }}$ of neutral $\mathrm{C}_{60}$. We derive a value of $1.6 \pm 0.1 \mathrm{eV}$ for the $E_{\mathrm{gap}}$ of neutral $\mathrm{C}_{60}$. We derive a value of $1.6 \pm 0.1 \mathrm{eV}$ for the $E_{\text {gap }}$ of $\mathrm{C}_{60}$, in qualitative agreement with the value of $1.9 \pm 0.5 \mathrm{eV}$ obtained in [13]. 


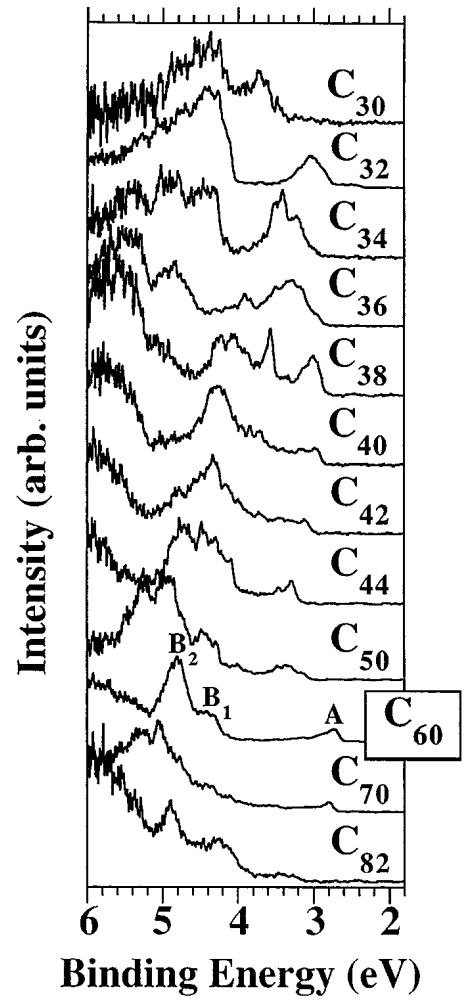

FIG. 2. Photoelectron spectra of strongly annealed fullerenes for a photon energy of $h \nu=6.4 \mathrm{eV}$. $A$ indicates the peak position of the LUMO; $B$ indicates the peak position of the $\mathrm{HOMO}$ of $\mathrm{C}_{60}$.

In some cases, the analysis of the photoelectron spectra and determination of $E_{\text {gap }}$ may be more complicated. Different isomers with different $E_{\text {gap }}$ may exist in the cluster beam. Then the relative intensities of their photoemission features change with the source conditions, which should allow them to be distinguished. Inelastic scattering of photoelectrons, multiplet splitting, and shakeup processes may lead to additional peaks. A broad peak may be an unresolved superposition of photoemission from several orbitals. Neglect of these effects leads to an enhanced uncertainty of the gap size in some cases, as indicated by the experimental error bars.

Given all of these factors, a determination of the gaps based solely on the experimental data is difficult A comparison to theoretical results allows a more reliable analysis of the spectra. Therefore we have performed calculations of the structure, energetics, and gap sizes of the fullerenes $C_{n}(n=30-82)$. The method we have applied is a density-functional-based tight-binding scheme (DF-TB). This method has been extensively described elsewhere [15]. Briefly we note that this scheme employs a nonorthogonal tight-binding model to determine the total energy and forces for a given atomic configu ration. In contrast to other tight-binding schemes, the Hamiltonian and overlap matrix elements are calculated from the firs principles. This method has already been successfully tested [15] and applied to fullerenes, see, e.g., Refs. $[16,17]$. The initial structures of the fullerenes, taken from [18], were optimized using efficien conjugate gradient algorithms. Since a systematic investigation of all possible cage structures, as performed for $\mathrm{C}_{40}$ [16], is unfeasible, we have considered only the structures of the so-called "fullerene road," as proposed by Fowler and Manolopoulos [18],

In Fig. 3 we display the experimental HOMO-LUMO gaps determined from the photoemission spectra and the calculated gaps and stabilities for the most stable isomers. We fin an outstanding correspondence between relative intensities in the mass spectrum and calculated stabilities $\delta$. Maxima of $\delta$ occur for the same clusters as maxima in abundances. $\mathrm{C}_{60}$ and $\mathrm{C}_{70}$, generally assumed to be the most stable fullerenes, exhibit the highest values of $\delta$. The small mass signal of $\mathrm{C}_{70}$ in Fig. 1 is due to the optimization of the source and annealing conditions for preferential production of small fullerenes. For $\mathrm{C}_{32}$, $\mathrm{C}_{36}, \mathrm{C}_{44}$, and $\mathrm{C}_{50}$, remarkably high $\delta$ are found, with definitel smaller values for adjacent fullerenes. This may be significan for an enhanced formation of these clusters. The high stabilities and extremely large mass signals support the theoretical predictions of these clusters as magic numbers.

The experimental gaps are in excellent agreement with the calculated values, except for $\mathrm{C}_{50}$. For $\mathrm{C}_{32}$ a remarkably large gap of $1.3 \mathrm{eV}$ is found experimentally and theoretically, indicating a low reactivity of the corresponding isomer. This gap is equal to the experimental gap of $\mathrm{C}_{70}\left(E_{\mathrm{gap}}=1.3 \mathrm{eV}\right)$ and not much smaller than the gap of $\mathrm{C}_{60}\left(E_{\mathrm{gap}}=1.6 \mathrm{eV}\right)$, which are both in accordance with former results $[13,18]$. Thus our results confir theoretical predictions of a large gap for $\mathrm{C}_{32}$ $[10,11,19,20]$. For $\mathrm{C}_{36}, \mathrm{C}_{44}$, and $\mathrm{C}_{50}$, large gaps of 0.8 , 0.8 , and $1 \mathrm{eV}$ are observed.

We fin a good correlation between experimental intensities, stabilities $\delta$, and HOMO-LUMO gaps for all

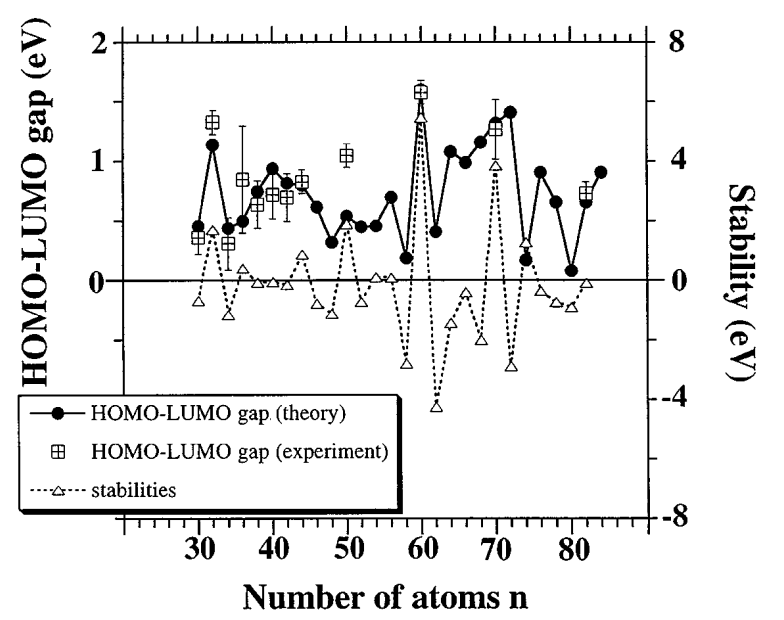

FIG. 3. Experimental (squares) and theoretical (dots) gaps and stabilities $\delta$ (triangles) of the fullerenes. 
of the fullerenes up to $n=70$. The theoretical values agree with calculations by Zhang et al. [10] obtained in a different scheme. For the firs time we could confir this correlation experimentally.

Our results suggest strongly that the most abundant fullerenes are those which exhibit both high stability and a large gap. This has also been pointed out in [10], but there it is assumed that the gap, governing the chemical reactivity, is only important for the isolation process. The absence of solvents in our experiments points out the importance of the gap for the formation process itself and the survival of the fullerenes.

For $\mathrm{C}_{60}$ and $\mathrm{C}_{70}$, pronounced maxima in $\delta$ correlate with large gaps. A comparable situation exists for $\mathrm{C}_{32}$ : Large intensity, high stability, and an anomalously large gap indicate high fragmentation stability and low reactivity. The direct neighbors $\mathrm{C}_{30}$ and $\mathrm{C}_{34}$ exhibit clearly lower stabilities and much smaller mass signals and gaps. Our results suggest that $\mathrm{C}_{32}$ is the most stable cluster below $\mathrm{C}_{60}$, in accordance with earlier theoretical predictions $[12,21]$. Thus it is a strong candidate for the formation of a fullerene material. Also, for $n=36,44$, and 50, considerably high mass intensities coincide with maxima in the calculated stabilities. The gaps are smaller than the gap of $\mathrm{C}_{32}$, but larger than the gaps of the other small fullerenes. $E_{\text {gap }}$ of $\mathrm{C}_{36}$ has a large experimental error bar and the calculations indicate the tendency for a smaller gap. For $\mathrm{C}_{50}$ a smaller than experimental gap has been predicted theoretically, and agreement is not improved by consideration of other isometric structures [18]. The large experimental gap of $\mathrm{C}_{50}$ indicates low reactivity in agreement with its high mass signal.

We have demonstrated that, in a laser vaporation source, high abundances of small fullerenes, especially of $\mathrm{C}_{32}$, can be achieved. We are convinced that, under appropriate source conditions, sufficien $\mathrm{C}_{32}$ for fullerite preparation can also be obtained in the soot of a furnace. The large gap of $1.3 \mathrm{eV}$ indicates a high stability of $\mathrm{C}_{32}$ against chemical reactions and spontaneous coalescence. Thus, presuming that a suitable method for the extraction and separation can be found, the synthesis of $\mathrm{C}_{32}$ fullerite should be possible. Additionally, $\mathrm{C}_{50}, \mathrm{C}_{44}$, and $\mathrm{C}_{36}$ are likely candidates for the formation of cluster materials.

This work was supported by SFB 341. R. R. acknowledges support by the Universität Köln within the Hochschulsonderprogramm III. P. W.F. and G. S. thank the British Council and the DAAD within the "BritishGerman Academic Research Collaboration Programme" for support.

Note added in proof.-Meanwhile, C. Piscoti, T. Yarger, and A. Zettl have published a paper claiming to have produced a solid consisting of $\mathrm{C}_{36}$ [Nature (London) 393, 771 (1998)].

[1] H. Handschuh, G. Ganteför, B. Kessler, P. S. Bechthold, and W. Eberhardt, Phys. Rev. Lett. 74, 1095 (1995).

[2] R. O. Jones and G. Seifert, Phys. Rev. Lett. 79, 443 (1997).

[3] S. C. O'Brien, J. R. Heath, R. F. Curl, and R. E. Smalley, J. Chem. Phys. 88, 220 (1988).

[4] M. Foltin, O. Echt, P. Scheier, B. Dünser, R. Wörgötter, D. Muigg, S. Matt, and T. D. Märk, J. Chem. Phys. 107, 6246 (1997); P.E. Barran, S. Firth, A. J. Stace, H. W. Kroto, K. Hansen, and E. E. B. Campbell, Int. J. Mass Spectrom. Ion Process 167/168, 127 (1997).

[5] H. Handschuh, G. Ganteför, and W. Eberhardt, Rev. Sci. Instrum. 66, 3838 (1995).

[6] Y.A. Yang, P. Xia, A. L. Junkin, and L. A. Bloomfield Phys. Rev. Lett. 66, 1205 (1991).

[7] E. A. Rohlfing D. M. Cox, and A. Kaldor, J. Chem. Phys. 18, 3322 (1984).

[8] H. W. Kroto, J.R. Heath, S.C. O'Brien, R. F. Curl, and R. E. Smalley, Nature (London) 318, 162 (1985).

[9] H. W. Kroto, Science 242, 1139 (1988).

[10] B. L. Zhang, C. H. Xu, C.Z. Wang, C. T. Chan, and K. M. Ho, Phys. Rev. B 46, 7333 (1992).

[11] D. E. Manolopoulos, J.C. May, and S.E. Down, Chem. Phys. Lett. 181, 105 (1991).

[12] P. W. Fowler, J. Phys. Chem. Solids 54, 1825 (1993).

[13] S. H. Yang, C.L. Pettiette, J. Conceicao, O. Cheshnovsky, and R.E. Smalley, Chem. Phys. Lett. 139, 233 (1987).

[14] Y. Wang, J. Phys. Chem. 96, 764 (1992).

[15] D. Porezag, Th. Frauenheim, Th. Köhler, G. Seifert, and R. Kaschner, Phys. Rev. B 51, 12947 (1995); G. Seifert, D. Porezag, and Th. Frauenheim, Int. J. Quantum Chem. 58, 185 (1996).

[16] P. W. Fowler, T. Heine, D. E. Manolopoulos, D. Mitchell, G. Orlandi, R. Schmidt, G. Seifert, and F. Zerbetto, J. Phys. Chem. 100, 6984 (1996).

[17] A. Ayuela, P. W. Fowler, D. Mitchell, R. Schmidt, G. Seifert, and F. Zerbetto, J. Phys. Chem. 100, 15634 (1996).

[18] P. W. Fowler and D. E. Manolopoulos, An Atlas of Fullerenes (Clarendon Press, Oxford, 1995).

[19] M.F. Fan, Z. Lin, and S. Yang, J. Mol. Struct. (Theochem.) 337, 231 (1995).

[20] N. Kurita, K. Kobayashi, H. Kumahora, K. Tago, and K. Ozawa, Chem. Phys. Lett. 188, 181 (1992).

[21] M. Manoharan, M. M. Balakrishnarajan, P. Venuvanalingam, and K. Balasubramanian, Chem. Phys. Lett. 222, 95 (1994). 